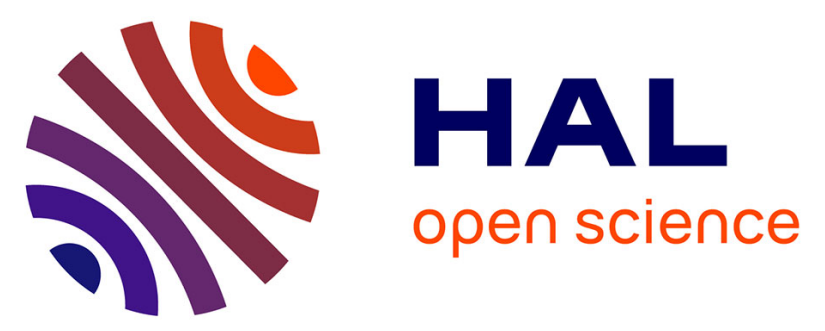

\title{
Elastic Shape Analysis of Cylindrical Surfaces for 3D/2D Registration in Endometrial Tissue Characterization
}

Chafik Samir, Sebastian Kurtek, Anuj Srivastava, Michel Canis

\section{To cite this version:}

Chafik Samir, Sebastian Kurtek, Anuj Srivastava, Michel Canis. Elastic Shape Analysis of Cylindrical Surfaces for 3D/2D Registration in Endometrial Tissue Characterization. IEEE Transactions on Medical Imaging, 2014, 33 (5), pp.1035 - 1043. 10.1109/TMI.2014.2300935 . hal-01598208

\section{HAL Id: hal-01598208 https://hal.science/hal-01598208}

Submitted on 16 Oct 2017

HAL is a multi-disciplinary open access archive for the deposit and dissemination of scientific research documents, whether they are published or not. The documents may come from teaching and research institutions in France or abroad, or from public or private research centers.
L'archive ouverte pluridisciplinaire HAL, est destinée au dépôt et à la diffusion de documents scientifiques de niveau recherche, publiés ou non, émanant des établissements d'enseignement et de recherche français ou étrangers, des laboratoires publics ou privés. 


\title{
Elastic Shape Analysis of Cylindrical Surfaces for 3D/2D Registration in Endometrial Tissue Characterization
}

\author{
Chafik Samir ${ }^{1}$, Sebastian Kurtek ${ }^{2}$, Anuj Srivastava ${ }^{3}$, and Michel Canis ${ }^{1}$ \\ ${ }^{1}$ ISIT UMR 6284 CNRS UdA, University of Clermont, France \\ ${ }^{2}$ Department of Statistics, Ohio State University, Columbus, OH, USA \\ ${ }^{3}$ Department of Statistics, Florida State University, Tallahassee, FL, USA
}

\begin{abstract}
We study the problem of joint registration and deformation analysis of endometrial tissue using 3D MRI and 2D Trans-vaginal Ultrasound (TVUS) measurements. In addition to the different imaging techniques involved in the two modalities, this problem is complicated due to: (1) different patient pose during MRI and TVUS observations, (2) the 3D nature of MRI and 2D nature of TVUS measurements, (3) the unknown intersecting plane for TVUS in MRI volume, and (4) the potential deformation of endometrial tissue during TVUS measurement process. Focusing on the shape of the tissue, we use expert manual segmentation of its boundaries in the two modalities and apply, with modification, recent developments in shape analysis of parametric surfaces to this problem. First, we extend the 2D TVUS curves to generalized cylindrical surfaces through replication, and then we compare them with MRI surfaces using elastic shape analysis. This shape analysis provides a simultaneous registration (optimal re-parameterization) and deformation (geodesic) between any two parametrized surfaces. Specifically, it provides optimal curves on MRI surfaces that match with the original TVUS curves. This framework results in an accurate quantification and localization of the deformable endometrial cells for radiologists, and growth characterization for gynecologists and obstetricians. We present experimental results using semi-synthetic data and real data from patients to illustrate these ideas.
\end{abstract}

Index Terms-Elastic geodesics, 3D-2D registration, elastic deformation, generalized cylinders, re-parametrization, shape analysis, endometriosis, multi-modal registration.

\section{INTRODUCTION}

Endometriosis is a complex gynecological medical disease in which endometrial cells (glands and stroma) appear outside their usual locations in the uterine cavity, as illustrated using Fig. I(a). The main symptoms of endometriosis may depend on the site of active endometriosis and are influenced by hormonal changes. This disease affects approximately $10 \%$ of women in the reproductive age group and may cause chronic pelvic pain, severe dysmenorrhea, and several digestive problems including diarrhea, occasional constipation, rectal bleeding and infertility. The endometriosis can be found in the pelvic cavity region, specifically in the pelvic peritoneum and pelvic organs. Currently there is no ethologic cure for endometriosis, but it can be treated in a variety of ways, including using pain medication, hormonal treatments, and laparoscopic surgery in severe cases.

As shown in Fig. I(a), the endometriosis tissue frequently penetrates into other tissues and organs. An accurate diagnosis must be made in order to obtain important medical information, such as the depth of infiltration and exact location. Several methods have been used for the purpose of assessing whether a patient has endometriosis. These methods use multiple imaging modalities, such as MRI, transvaginal ultrasound (TVUS), pelvic ultrasound, and laparoscopy to determine the stage of endometriosis in a patient. If MRI and ultrasound techniques fail to detect endometrial lesions, then laparoscopy becomes necessary. Laparoscopy involves minor surgery under general anesthesia and, for many years, laparoscopy was the only method to diagnose endometriosis [1]. However, there are some limitations in this approach. First, it does not provide any

Manuscript received xxx; revised xxx. information about the depth of infiltration. Second, it might not detect the endometrial lesions which are located under other organs. Finally, the place of insertion of the laparoscope into the patient's body depends on the prediction of the location of endometriosis by gynecologists. To avoid these drawbacks, an attractive solution is to combine information collected from TVUS and MR imaging and reach a comprehensive inference on endometrial lesions.

The MRI provides a global map of a nodule's location and gives a good contrast between normal and malignant tissues, as shown in Fig. I(c). However, MR imaging does not include information about the elasticity of endometrial lesions [1]. On the other hand, TVUS imaging can provide information about the depth of infiltration and, importantly, about the tissue deformation resulting from the transducer's pressure. An example of a TVUS image is shown in Fig. I(b). The resulting nonlinear deformation is highly dependent on the transducer's position, area, shape, and pressure. This scheme of imaging is depicted in Fig. 2 where a surface (shown in the left) is observed using a transducer that changes the surface shape locally (in the middle) and captures a planar image shown in the right. The limitations of TVUS imaging include limited field of view and low signal to noise ratio [2]. Due to their different operating principles, TVUS and MRI can provide complementary information for diagnosing endometriosis. In order to counter the individual limitations of MR and TVUS imaging modalities, the registration and fusion of MR/TVUS data must be performed. The main advantage of MR/TVUS fusion is the combination of accurate localization (from MR) and estimation of the depth of infiltration (from TVUS), which can help the surgeon accurately remove pathological tissues. However, registration of this imaging data across modalities is 


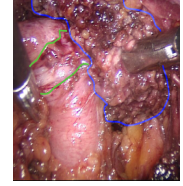

(a) Tissues

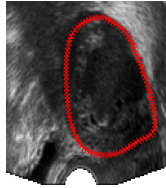

(b) TVUS

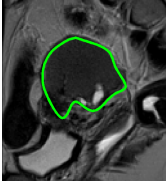

(c) MRI

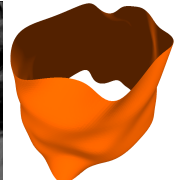

(d) Surface
Fig. 1. Example of endometrial tissue localization outside the pelvic cavity shown in laparoscopic (a), TVUS (b), and MR (c) images.

challenging due to differences in the tissue's intensity, dimensionality, patient positioning (translation, rotation, and scaling) and the organ's elasticity (re-parametrization) introduced by the transducer's pressure. Furthermore, registration and fusion of these two images is a challenging task due to the disparity in gray-level intensities, the existence of artifacts including deformation and noise, and the large non-overlapping fields of view. Therefore, shape analysis of structures becomes an important tool in this context. Before we describe our shapebased approach to registration of the two modalities, we summarize related research from past literature.

\section{A. Related Work}

The general problem of registering 2D or/and 3D images is especially hard because: (1) often data are derived from different modalities, (2) they represent deformable organs with different shapes in different images, and (3) there is no initial correspondence between shapes contained therein. The problem of 3D-2D registration is a special case of the general image registration problem, with a high interest to clinicians. In a radiological context, the precise knowledge of position and elasticity of some specific organs is very important. With the help of 3D-2D registration methods the surgeon can obtain such knowledge, and benefit in time efficiency and safety during the laparoscopic intervention. In this section, we provide a short summary of some relevant works in 3D-2D registration in the context of diagnosis, treatment, and guided-intervention. A large majority of existing ideas are concerned with registering pre-operative data such as MRI, CT, MR Angiography (MRA), Digital Subtracted Angiography (DSA), etc., to the inter-operative data, i.e. data available during the surgery. Although our problem context is different, some of these methods are relevant as they have the same goal, i.e. register a $3 \mathrm{D}$ object to a $2 \mathrm{D}$ one. Based on the representations used for registration, one can roughly categorize the most common approaches into feature-based and intensity-based.

Intensity-based methods: Intensity-based registration methods are quite common in medical image analysis as they can use pixel/voxel values to register single or multiple modalities. Most of the intensity-based methods consist of minimizing a cost function with two terms: (1) a data term that consists of one of the following measures: mutual information, entropy, cross correlation or others, and (2) a regularization term that represents a regularization of the deformation of the first or second order [3]. A common approach is to integrate similarity metrics to match image representations. There are two classes of such methods: first, images are represented by their intensity distribution on the whole images [4] or on some characteristic subregions [5]. This is the case when a surface is deformed using a parametric model to simulate mammographic compression [6], or when a probabilistic model is defined to estimate a cutting plane as a registration between 3D MRAs and 2D X-ray DSAs [7]. The other types of methods represent both images in an artificial modality using morphological tools [8], color variances [9], gradient directions [10], etc. The main drawback of methods of the first type is that they are highly dependent on the images' intensity correlation. A big difference between intensity values, such as in TVUS and MRI of endometriosis, can make those approaches prone to large errors. Methods of the second type are even more challenging for TVUS images, which vary significantly in their appearance due to multiple echoes and repetition times.

Feature-based methods: Feature-based methods are those that make use of landmarks or other extracted geometric features to register images. Such methods rely on lowdimensional transformations, such as rigid or affine, for registration. For example, Cosse et al. [11] proposed an ICP-based algorithm to register MR/US prostate and rectum surfaces. Vermandel et al. [12] used a hybrid approach by combining both intensity and skeleton-based methods to register 3D MRAs and 2D DSAs. The registration process consists of estimating the six (rigid-body) motion parameters between the 2D image and the projected views of the 3D structure. More recently, Biesdorf et al. [13] used a combination of 3D modelbased segmentation and elastic image registration to quantify the aortic arch. Their method consists of minimizing an energy functional corresponding to a segmented 3D cylindrical tubular model as a first term and a 2D registered circular cross-section as a second term. However, they used a parametric spline model to ensure smoothness of the resulting displacement field, which makes the method lose its generality in function spaces. Although this paper has a similar setting to the current paper, the focus is somewhat different. In that paper, one assumed the knowledge of projection from 3D to $2 \mathrm{D}$ and used this information to segment a $3 \mathrm{D}$ volume. In our case, the cutting plane for TVUS is unknown and the projection is not available. Furthermore, we have an unknown deformation between the 3D and 2D objects. We assume the availability of segmentation to estimate the cutting plane, the registration, and the deformation between objects.

Some of the current approaches consider registration using locally-affine transformations. Here, one splits the deformation vector field into small regions. For example, Zikic et al. [14] used Markov random fields to estimate affine transformations that align $3 \mathrm{D}$ to $2 \mathrm{D}$ data by comparing the $2 \mathrm{D}$ projected views of the volume and the available $2 \mathrm{D}$ images. Also, this way the deformations can be computed locally and then interpolated. Buerger et al. [15] exploited the same idea by using an adaptive region-based method to estimate wholethorax deformations during a respiratory motion. They split the $3 \mathrm{D}$ volume into hierarchical structures including adaptive sub-blocks. A local affine transformation is then estimated 
on each block and a multilevel B-spline interpolation is used to estimate a full displacement (a deformation field on the whole volume). To cope with the limitations of intensitybased approaches, Zhang et al. [16] combine intensity and uncertainty weighted surfaces to register 3D CBCT and 3D FBCT prostate images. Their cost function includes three terms: the similarity between images, the similarity between spherical surfaces including uncertainty, and the regularization term.

Among all intensity or hybrid based techniques, the methods relying on mutual information have been shown to robustly align images from different imaging modalities. However, matching two image distributions assumes that they are sufficiently correlated which is not the case when images are produced from very distinct modalities, resulting in different data dimensions.

\section{B. Our Approach}

Since the main goal of this paper is to study endometriosis, we focus on this anatomical structure directly. The purpose of this work is to characterize endometrial tissues in MR images and, since the nodules can be deformed in the TVUS images, there is no automatic correspondence between features in both modalities. Thus, we propose a new method based on shape analysis, since a shape feature is invariant to translation, scale, rotation, and re-parametrization. We assume that we can extract, manually or automatically, the boundaries of endometrial tissue in MRI and TVUS images. In case of 3D MRI images, we obtain a 2D surface as the boundary, while for TVUS images we get a simple closed curve. Although the boundary of an endometrium forms a closed surface, the MRI image is collected at such a low resolution that in the absence of the closing slices on both ends, the resulting surfaces are topologically cylindrical. So, our modified goal is now to focus on the shapes of these two objects - a cylindrically-parameterized surface from MRI and a planar closed curve from TVUS - and perform registration of these extracted structures. When comparing two objects in different dimensions, as is the case here, there are two possibilities. One is to project the higher-dimensional object to the lower space and then compare with the other object. However, the parameters of projection (or the plane of intersection) are unknown and can make a big difference in the final result. The other possibility is to extrapolate the lower-dimensional object to the higher space and perform a more exhaustive comparison Although this approach can be computationally expensive, it is more stable with respect to the choice of parameters. So, we will take this approach by extending the TVUS curve into a generalized cylinder and comparing the two shapes. In this setup, we pose the following questions:

1) Which closed curve on the MRI surface optimally corresponds to the TVUS curve?

2) What is the optimal deformation between the two curves and how to quantify the elasticity of endometriosis using a measure of this deformation?

3) Can we extend this deformation from the optimal curve to the full MRI surface to help a physician in locating and assessing endometrium shape variability?
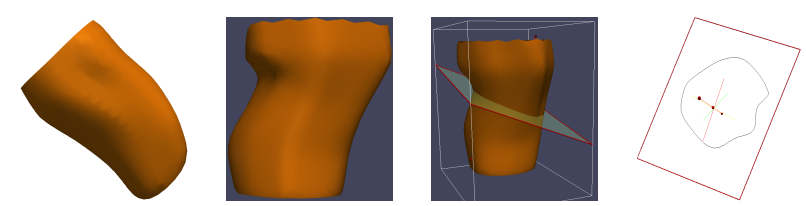

Fig. 2. An illustration of the mapping from an MRI surface $f_{M R I}$ to the TVUS curve $\beta_{U S}$. Left: an open cylinder depicting an MRI surface $f_{M R I}$. Middle: $\Phi\left(T\left(f_{M R I}\right)\right)$ after a nonlinear deformation $\Phi$ and its intersection with a cutting plane. Right: Resulting in $\beta_{U S}$.

To answer these and related questions, we propose a surfacebased elastic registration and deformation method for this task. This approach is a modification of shape analysis of certain parametric surfaces developed by Kurtek et al. [17], [18] in previous papers. While previous papers dealt with spherical and quadrilateral surfaces, the current paper will extend that mathematical framework to cylindrically-parameterized surfaces. The main strength of this framework is that it solves the problems of registration and optimal deformation in a joint framework.

We summarize the goal of this paper in precise mathematical form. As stated above, the variability between TVUS and MRI images results from two transformations. Firstly, there is a change in patient position due to the fact that MR imaging is performed in supine position, whilst TVUS imaging is performed in lithotomy position. As shown in Fig. 2, the first transformation, denoted by $T$, introduces a change in translation and rotation of the 3D MRI surface $f_{M R I}$ (depicted as a cylinder). Secondly, there is a nonlinear deformation $\Phi$ due to the ultrasound transducer's pressure, resulting in the deformed surface $\Phi\left(T\left(f_{M R I}\right)\right)$. Thus, a planar curve on the MRI surface may be transformed to a non-planar one on $\Phi\left(T\left(f_{M R I}\right)\right)$, and vice versa. Let $\beta_{U S}$ be a planar curve observed using the intersection of a cutting plane $P$ with $\Phi\left(T\left(f_{M R I}\right)\right)$, i.e. $\beta_{U S}=P \cap \Phi\left(T\left(f_{M R I}\right)\right)$. The problem of interest is: Given $\beta_{U S}$ and $f_{M R I}$, estimate a closed curve $\hat{\beta}_{M R I} \in f_{M R I}$ that optimally corresponds to $\beta_{U S}$ under elastic deformations. Note that $T, \Phi$, and $P$ are unknown nuisance variables and one needs to estimate them, at least partially, in the estimation process.

The rest of the paper is organized as follows. We describe a conceptual overview of our framework in Section 2 and the mathematical framework for registration of cylindricallyparametrized surfaces in Section 3. We use that framework to compute optimal deformations (geodesics) between surfaces in Section 4. The application of this framework to analyze MRI surfaces and TVUS curves is presented in Section 5, with some validation analysis in Section 6. We close this paper with a brief conclusion in Section 7.

\section{MATHEMATICAL Framework}

Let $\beta_{U S}: \mathbb{S}^{1} \rightarrow \mathbb{R}^{2}$ represent a closed, planar curve coming from the TVUS image. Also, let $f_{M R I}: \mathbb{S}^{1} \times[0,1] \rightarrow \mathbb{R}^{3}$ be the surface obtained from the MRI image. We will develop a novel Riemannian framework for shape analysis of cylindrically-parametrized surfaces. In particular, the developed tools will give us a principled approach for registra- 
tion and deformation of surfaces. Furthermore, the resulting distance (that satisfies symmetry and triangle inequality) will be based on an elastic metric, which explicitly captures the amount of "stretching" and "bending" needed to deform one surface into another. In order to use this framework, we form an ultrasound surface using $f_{U S}: \mathbb{S}^{1} \times[0,1] \rightarrow \mathbb{R}^{3}$ by replicating $\beta_{U S}$ in the axial direction, i.e. $f_{U S}(u, v)=\beta_{U S}(u), \forall v \in$ $[0,1]$. This surface represents all of the information provided by $\beta_{U S}$ coming from the ultrasound image. Our approach will be to compare shapes of $f_{U S}$ and $f_{M R I}$ in an elastic framework. This requires the definition of the elastic metric and a set of tools to register and compute geodesics (and geodesic distances) between surfaces. We present these details next.

\section{A. Representation of Surfaces}

Let $\mathcal{F}$ be the space of all smooth embeddings of a cylinder in $\mathbb{R}^{3}$ and let $\Gamma$ be the set of all boundary-preserving diffeomorphisms from $\mathbb{S}^{1} \times[0,1]$ to itself. It acts naturally on $\mathcal{F}$ by composition: $(f, \gamma)=f \circ \gamma$, where $\circ$ represents the composition of $f$ and $\gamma$. In other words, it is a reparametrization of the surface $f$ using $\gamma$. Since $\mathcal{F}$ is a vector space, the tangent space at $f \in \mathcal{F}, T_{f}(\mathcal{F})$, is $\mathcal{F}$ itself. Using two tangent vectors $v_{1}, v_{2} \in T_{f}(\mathcal{F})$ we can define the standard inner product on $\mathcal{F}$ as follows $\left\langle v_{1}, v_{2}\right\rangle=$ $\int_{\mathbb{S}^{1} \times[0,1]}\left\langle v_{1}(s), v_{2}(s)\right\rangle d s$, where $d s$ is the standard Lebesgue measure on $\mathbb{S}^{1} \times[0,1]$ and the $\mathbb{L}^{2}$ norm between any two surfaces $f_{1}, f_{2} \in \mathcal{F}$ is $\left(\int_{\mathbb{S}^{1} \times[0,1]}\left|f_{1}(s)-f_{2}(s)\right|^{2} d s\right)^{\frac{1}{2}}$. As was previously shown in many works ([19], [20], [18], [17]), such a framework is not appropriate for analyzing shapes of surfaces because the action of $\Gamma$ does not preserve distances, i.e. $\left\|f_{1}-f_{2}\right\| \neq\left\|f_{1} \circ \gamma-f_{2} \circ \gamma\right\|$. One solution is to restrict the analysis to area-preserving diffeomorphisms [21], but this is very limiting in practice. Another approach, taken by [19], [20], [18], [17], is to utilize a different representation of surfaces such that the action of $\Gamma$ preserves $\mathbb{L}^{2}$ distances. Such representations are motivated by Riemannian metrics that: (1) make the action of $\Gamma$ on $\mathcal{F}$ by isometries, and (2) help quantify deformations needed to transform surfaces into each other. Jermyn et al. [19] defined a specific Riemannian metric and the corresponding mathematical representation to perform registration of spherical and quadrilateral surfaces; in this framework, the registration of points across surfaces is accomplished by re-parameterization of surfaces. The use of this metric is very intuitive in our application as one of our goals is to define a measure of elasticity of the ultrasound surface. We extend their work by considering cylindricallyparametrized surfaces, and in addition, we define a numerical technique termed path-straightening to compute geodesic paths and distances in $\mathcal{F}$ similar to [17].

Let $n(s)=\frac{\partial f}{\partial u}(s) \times \frac{\partial f}{\partial v}(s) \in \mathbb{R}^{3}$ denote the normal vector to the surface at the point $s=(u, v) \in \mathbb{S}^{1} \times[0,1]$. Using $n(s)$, we define a mathematical representation of surfaces termed square-root normal fields (SRNFs) [19].

Definition 1. For a surface $f: \mathbb{S}^{1} \times[0,1] \rightarrow \mathbb{R}^{3}$ define a mapping $Q: \mathcal{F} \rightarrow \mathbb{L}^{2}$ and the square root normal field (SRNF) $q: \mathbb{S}^{1} \times[0,1] \rightarrow \mathbb{R}^{3}$ as $Q(f)(s)=q(s)=\frac{n(s)}{\sqrt{|n(s)|}}$.

The $\mathbb{L}^{2}$-norm of $q$ equals the surface area of $f$ and thus the space of SRNFs is a subset of $\mathbb{L}^{2}\left(\mathbb{S}^{1} \times[0,1], \mathbb{R}^{3}\right)$ from now on referred to as $\mathbb{L}^{2}$. This representation of surfaces has a number of important properties:

1) As shown in [19], a complicated elastic metric on $\mathcal{F}$ simplifies to the standard $\mathbb{L}^{2}$ metric on the space of SRNFs. This greatly simplifies the development of certain shape analysis tools such as registration and deformation. It also provides explicit interpretations of the types of deformations measured by the distance function introduced later.

2) The SRNF $q$ does not depend on a translation of $f$, since it only depends on spatial derivatives of $f$.

3) $S O(3)$ is the special orthogonal group, which contains all $3 \times 3$ rotation matrices. For $O \in S O(3)$ and $f \in \mathcal{F}$, the SRNF of a rotated surface $O f$ is $O q . \Gamma$ is the reparameterization group. For a $\gamma \in \Gamma$ and $f \in \mathcal{F}$, the SRNF of a re-parameterized surface $f \circ \gamma$ is $(q, \gamma)=$ $(q \circ \gamma) \sqrt{J_{\gamma}}$, where $J_{\gamma}$ is the determinant of the Jacobian of $\gamma$. Furthermore, the actions of $S O(3)$ and $\Gamma$ on $\mathbb{L}^{2}$ commute.

4) The action of the product group $S O(3) \times \Gamma$ on $\mathbb{L}^{2}$ is by isometries. In other words, if we rotate and reparametrize any two surfaces in the same way, the $\mathbb{L}^{2}$ norm between their SRNFs remains unchanged: $\| q_{1}-$ $q_{2}\|=\|\left(O q_{1}, \gamma\right)-\left(O q_{2}, \gamma\right) \|[19]$.

Since our notion of shape is invariant to translation, scale, rotation and re-parameterization, we need to ensure these invariances. The SRNFs are already invariant to translations and we can achieve scale invariance by re-scaling all surfaces to have unit surface area. The re-sclaling is problematic in our application because we do not know the relative scale of $\beta_{U S}$ and the third coordinate in $f_{U S}$. Thus, we take a different approach. We re-scale all of the surface coordinates of $f_{U S}$ individually to lie in $[0,1]$. In order to define a rotation and parameterization invariant distance on $\mathbb{L}^{2}$ we first define an equivalence class of an SRNF as all possible rotations and re-parameterizations of this function: $[q]=\{(O q, \gamma) \mid O \in$ $S O(3), \gamma \in \Gamma\}$. The distance between shapes of surfaces is then given by:

$$
d\left(\left[q_{1}\right],\left[q_{2}\right]\right)=\inf _{(O, \gamma) \in S O(3) \times \Gamma}\left\|q_{1}-\left(O q_{2}, \gamma\right)\right\| .
$$

Note that while this distance is important in quantifying differences between shapes of surfaces it defines an extrinsic distance. It does not provide a recipe for computing geodesic paths between surfaces due to difficulties in computing the inverse mapping of $Q$.

It is also important to point out that the distance in Eqn. 1 is fundamentally different from the distance proposed in [18]. The distance in [18] is based on a different representation of surfaces termed the $q$-map (defined as $q(s)=\sqrt{|n(s)|} f(s)$ ). The advantages of the SRNF representation compared to the $q$-map representation are described in more detail in [19]. 

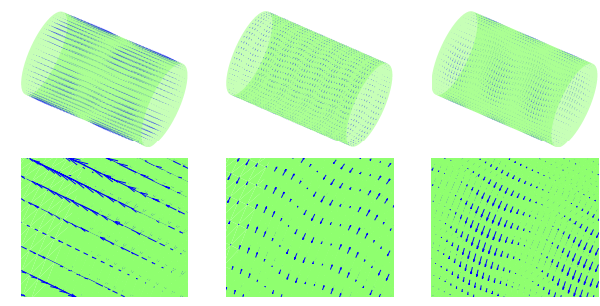

Fig. 3. Examples of basis elements of $T_{\gamma_{i d}}(\Gamma)$ that are used in incremental optimization over $\Gamma$. For each example we display the full vector field (top) and a zoom-in on a specific part (bottom).

\section{B. Registration of Cylindrical Surfaces}

The computation of $d$ in Eqn. 1 requires solving the joint optimization problem on $S O(3) \times \Gamma$. This procedure, albeit with different mathematical representations of surfaces, has been previously provided in [20], [18], [19] for different parameter domains (unit sphere, unit square, etc.) and we give a brief summary next for convenience. The novelty of this paper lies in extending these methods to the case of the cylindrical domain $\mathbb{S}^{1} \times[0,1]$.

For a fixed $\gamma \in \Gamma$, the minimization over $S O(3)$ can be easily performed directly using Procrustes analysis. Let $\tilde{q}_{2}$ denote $\left(q_{2}, \gamma\right)$; then, the optimal value of $O$ is obtained as follows. Compute the $3 \times 3$ matrix $A=\int_{\mathbb{S}^{1} \times[0,1]} q_{1}(s) \tilde{q}_{2}(s)^{T} d s$. Using singular value decomposition $A=U \Sigma V^{T}$, we can define the optimal rotation as $O^{*}=U V^{T}$ (if the determinant of $A$ is negative, the last column of $V$ changes sign).

In order to solve the optimization problem over $\Gamma$ (for a fixed rotation), we will use a gradient approach. To specify the gradient for iterative updates, we define the cost function at any iteration to be: $E: \Gamma \rightarrow \mathbb{R}, E[\gamma]=\left\|q_{1}-\left(q_{2}, \gamma\right)\right\|^{2}=$ $\left\|q_{1}-\phi(\gamma)\right\|^{2}$, where $\phi: \Gamma \rightarrow\left[q_{2}\right]$ is defined to be $\phi(\gamma)=$ $\left(q_{2}, \gamma\right)$. Let $b$ be a unit vector in $T_{\gamma_{i d}}(\Gamma)$ for $\gamma_{i d}(s)=s$. The tangent space $T_{\gamma_{i d}}(\Gamma)$ is the set of all smooth vector fields tangent to the cylinder with appropriate boundary conditions. Using an orthonormal basis for $T_{\gamma_{i d}}(\Gamma)$ we can compute the full gradient of $E$ at $\gamma_{i d}$ and use it to update the current estimate of the optimal parameterization of $q_{2}$. In principle, this tangent space is infinite dimensional, but we restrict to a finite number of basis elements. This basis is formed by considering the coordinates on $\mathbb{S}^{1}$ and $[0,1]$ separately. Tangent vector fields on $\mathbb{S}^{1}$ can be written as $\left\{\sin \left(k_{1} u\right), 1-\right.$ $\left.\cos \left(k_{1} u\right), u, 1-u \mid k_{1}=1, \ldots, n_{1}, u \in \mathbb{S}^{1}\right\}$. Tangent vector fields on $[0,1]$ can be written as $\left\{\sin \left(2 \pi k_{2} v\right), 1-\right.$ $\left.\cos \left(2 \pi k_{2} v\right) \mid k_{2}=1, \ldots, n_{2}, v \in[0,1]\right\}$. We take all possible products of the elements of these two sets and orthonormalize them using Gram-Schmidt. Similar basis construction for the sphere and unit square domains is described in [18], [20]. Some basis elements (vector fields on the cylinder) are shown in Fig. 3. With a basis of $T_{\gamma_{i d}}(\Gamma)$, the directional derivative of $E$ at $\gamma_{i d}$, in the direction of $b$, is given by $\left\langle q_{1}-\phi\left(\gamma_{i d}\right), \phi_{*}(b)\right\rangle b$, where $\phi_{*}: T_{\gamma_{i d}}(\Gamma) \rightarrow T_{q_{2}}\left(\left[q_{2}\right]\right)$ is the differential of $\phi$. (An expression for $\phi_{*}$ can be found in [18]. ) Furthermore, the full gradient can be approximated using $\sum_{b}\left\langle q_{1}-\phi\left(\gamma_{i d}\right), \phi_{*}(b)\right\rangle b$.

To solve the joint minimization problem in Eqn. 1, we alternate between minimizing over $S O(3)$ and $\Gamma$ and stop

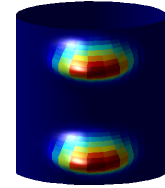

$f_{1}$

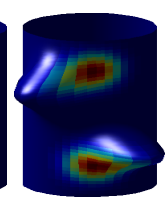

$f_{2}$

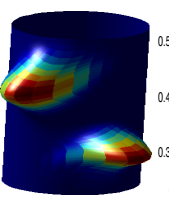

$f_{2} \circ \gamma^{*}$

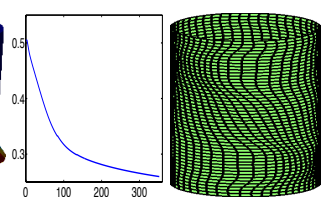

$\operatorname{cost}$

$\gamma^{*}$
Fig. 4. Using identical colors to denote registration, the figure shows improvement in registration of similar features across the two surfaces during optimization over $\Gamma$.

when we converge to a solution. Fig. 4 shows an example of this optimization for two surfaces - these surfaces have two bumps each but the bumps are placed at different points along the axial coordinate. The final registration provides a better matching of features, as depicted using identical color maps, across the two surfaces. The importance of registration is further emphasized later in the bottom row of Fig. 5 where we show geodesic paths between the same two surfaces, before and after the registration.

\section{Geodesics Between Cylindrical Surfaces}

As mentioned earlier, it is not a simple task to compute the inverse mapping of $Q$. This prevents us from computing the geodesic between surfaces by simply mapping the straight line path from the space of SRNFs to $\mathcal{F}$. For this reason, we develop a numerical technique, termed path-straightening, to compute geodesic paths and geodesic distances (these will now be intrinsic distances between surfaces) directly in $\mathcal{F}$. We will do this using the pullback metric from $\mathbb{L}^{2}$.

In order to define the pullback metric on $\mathcal{F}$ we begin by defining the differential of $Q, Q_{*}: T_{f}(\mathcal{F}) \rightarrow T_{Q(f)}\left(\mathbb{L}^{2}\right)$ (for $\left.w \in T_{f}(\mathcal{F})\right)$ :

$$
\begin{array}{r}
Q_{*, f}(w)(s)=\left.\frac{d}{d \epsilon} Q(f+\epsilon w)(s)\right|_{\epsilon=0} \\
=\frac{n_{w}(s)}{\sqrt{|n(s)|}}-\frac{\left(n(s) \cdot n_{w} s\right) n(s)}{2|n(s)|^{5 / 2}},
\end{array}
$$

where $n_{w}(s)=\frac{\partial f}{\partial u}(s) \times \frac{\partial w}{\partial v}(s)+\frac{\partial w}{\partial u}(s) \times \frac{\partial f}{\partial v}(s)$. Note that $w$ represents a vector field on the surface $f$. Using this expression, we can define a Riemannian metric on $\mathcal{F}$ for $w_{1}, w_{2} \in T_{f}(\mathcal{F})$ as follows:

$$
\begin{aligned}
& \left\langle\left\langle w_{1}, w_{2}\right\rangle\right\rangle_{f}=\left\langle Q_{*, f}\left(w_{1}\right), Q_{*, f}\left(w_{2}\right)\right\rangle= \\
& \quad \int \frac{\left(n_{w_{1}}(s) \cdot n_{w_{2}}(s)\right)}{|n(s)|}-\frac{3}{4} \int \frac{\left(n(s) \cdot n_{w_{1}}(s)\right)\left(n(s) \cdot n_{w_{2}}(s)\right)}{|n(s)|^{3}}
\end{aligned}
$$

In order to compute a geodesic path between two given surfaces $f_{1}, f_{2} \in \mathcal{F}$, we begin by defining the length of a path given by $F:[0,1] \rightarrow \mathcal{F}, F(0)=f_{1}, F(1)=f_{2}$ using the pullback metric (all arguments have been suppressed for brevity):

$$
\begin{aligned}
L(F)=\int_{0}^{1} & \sqrt{\left\langle\left\langle F_{t}, F_{t}\right\rangle\right\rangle_{F}} d t=\int_{0}^{1} \sqrt{\left\langle Q_{*, F}\left(F_{t}\right), Q_{*, F}\left(F_{t}\right)\right\rangle} d t \\
& =\int_{0}^{1} \sqrt{\int_{[0,1] \times \mathbb{S}^{1}}\left[\frac{-3\left(N \cdot N_{t}\right)^{2}}{4|N|^{3}}+\frac{\left|N_{t}\right|^{2}}{|N|}\right] d s d t .}
\end{aligned}
$$

In the above expression, we use $N(t)$ to denote $\frac{\partial F(t)}{\partial u}(s) \times$ $\frac{\partial F(t)}{\partial v}(s) . N(t)$ is a normal vector field on the surface $f=$ 
$F(t)$. Also, we denote partial derivatives using subscripts (i.e. $N_{t}$ ). It is a well known result that a critical point of this path energy provides a geodesic between $f_{1}$ and $f_{2}$ in $\mathcal{F}$. Equivalently, one can consider the following energy, which is computationally easier to work with:

$$
\begin{array}{r}
\tilde{E}(F)=\int_{0}^{1}\left\langle\left\langle F_{t}, F_{t}\right\rangle\right\rangle_{F} d t=\int_{0}^{1}\left\langle Q_{*, F}\left(F_{t}\right), Q_{*, F}\left(F_{t}\right)\right\rangle d t \\
=\int_{0}^{1} \int_{[0,1] \times \mathbb{S}^{1}}\left[\frac{-3\left(N \cdot N_{t}\right)^{2}}{4|N|^{3}}+\frac{\left|N_{t}\right|^{2}}{|N|}\right] d s d t
\end{array}
$$

Thus, a geodesic path between two surfaces $f_{1}$ and $f_{2}$ is the solution to the following minimization problem:

$$
F^{*}=\underset{F:[0,1] \rightarrow \mathcal{F}, F(0)=f_{1}, F(1)=f_{2}}{\operatorname{argmin}} \tilde{E}(F) .
$$

We will compute the solution to this problem using a gradient descent approach. We will approximate $\nabla \tilde{E}$ using directional derivatives $\nabla \tilde{E}_{F}(G)$ where $G \in \mathcal{G}$ and $\mathcal{G}$ is an orthonormal basis that spans all possible perturbations of a path $F$. We begin by computing $\nabla \tilde{E}_{F}(G)$ :

$$
\begin{aligned}
& \nabla \tilde{E}_{F}(G)=\left.\frac{d}{d \epsilon} \tilde{E}(F+\epsilon G)\right|_{\epsilon=0}= \\
& \iint\left[\frac{-3\left(N \cdot \delta_{G} N_{t}+N_{t} \cdot \delta_{G} N\right)\left(N \cdot N_{t}\right)-2\left|N_{t}\right|^{2}\left(N \cdot \delta_{G} N\right)}{2|N|^{3}}\right. \\
& \left.+\frac{9\left(N \cdot \delta_{G} N\right)\left(N \cdot N_{t}\right)^{2}}{4|N|^{5}}+\frac{2\left(N_{t} \cdot \delta_{G} N_{t}\right)}{|N|}\right] d s d t,
\end{aligned}
$$

where $\delta_{G} N=G_{u} \times F_{v}+F_{u} \times G_{v}$ and $\delta_{G} N_{t}=G_{t u} \times F_{v}+$ $F_{t u} \times G_{v}+G_{u} \times F_{t v}+F_{u} \times G_{t v}$ (subscripts are used for time and spatial derivatives here). Then, in order to approximate the gradient we will use a finite number of basis elements $G_{i}, i=1, \ldots, B: \nabla \tilde{E} \approx \sum_{i=1}^{B} \nabla \tilde{E}_{F}\left(G_{i}\right) G_{i}$. We will update the current estimate of the geodesic path according to $F=$ $F-\eta \nabla \tilde{E}$, where $\eta>0$ is a small step size. Once the algorithm has converged, it results in the geodesic path $\left(F^{*}\right)$ as well as the unique shooting vector field $\left(F_{t}(0)\right)$, which can be used to optimally deform $f_{1}$ to $f_{2}$ under our metric.

The basis set $\left\{G_{i}\right\}$ is created as follows. Each element $G:\left(\mathbb{S}^{1} \times[0,1]\right) \times[0,1] \rightarrow \mathbb{R}^{3}$ has three arguments $u, v, t$ where $(u, v)$ are the spatial coordinates on $\mathbb{S}^{1} \times[0,1]$ and $t$ is the time index along the path. We first form bases for each of the $u, v, t$ components separately: $P^{1}(u)=\{\sin (i u), \cos (i u)-$ $\left.1, u, 1-u \mid i=1, \ldots, n_{1}, \quad u \in \mathbb{S}^{1}\right\}, P^{2}(v)=$ $\left\{\sin (2 \pi j v), \cos (2 \pi j v), 1 \mid j=1, \ldots, n_{2}, v \in[0,1]\right\}, P^{3}(t)=$ $\left\{\sin (2 \pi k t), \cos (2 \pi k t)-1 \mid k=1, \ldots, n_{3}, t \in[0,1]\right\}$. In order to define the basis $\tilde{P}:\left(\mathbb{S}^{1} \times[0,1]\right) \times[0,1] \rightarrow \mathbb{R}$ we utilize all possible products of the three component bases $P^{1}(u)$, $P^{2}(v)$ and $P^{3}(t)$. The final step in forming the full basis is to utilize a copy of $\tilde{P}$ for each of the Euclidean coordinates (in $\mathbb{R}^{3}$ ) and to orthonormalize this basis using the Gram-Schmidt procedure.

Fig. 5 shows examples of minimization of $\tilde{E}$ using path straightening between surfaces before (top) and after (bottom) registration. Fig. 6 shows a geodesic between two cylindrically-parametrized surfaces $f_{1}$ and $f_{2}$ at the end points and uniformly spaced samples along the optimal path $F^{*}$.

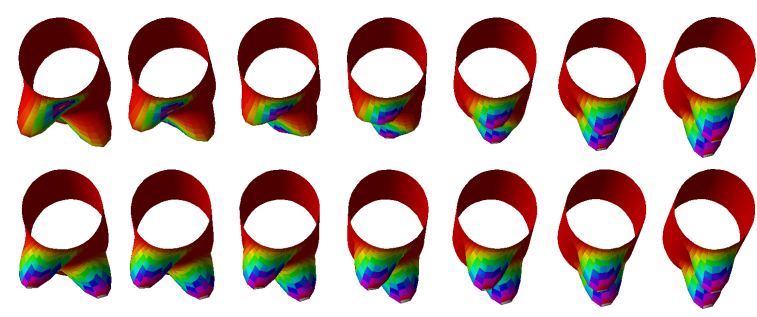

Fig. 5. Geodesic computation using path straightening between surfaces before (top) and after (bottom) registration.

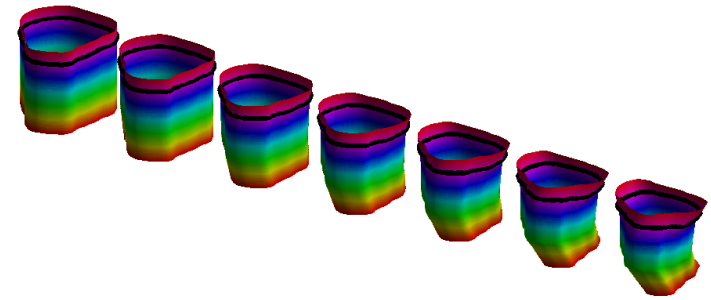

Fig. 6. Geodesic between two cylindrically-parametrized surfaces.

\section{ApPlication to 2D-3D REgistration}

Now that we have laid out tools for registering and deforming cylindrically-parameterized surfaces, we return to the four major goals outlined earlier and show how they are achieved using this framework. Starting with a surface $f_{M R I}$ and a curve $\beta_{U S}$, we first form a generalized cylinder $f_{U S}$ from $\beta_{U S}$. Then, we perform a registration of $f_{U S}$ and $f_{M R I}$ using the framework outlined in Sections II-A and II-B. Given this optimal registration, we compute the geodesic distance and geodesic path between these surfaces using tools developed in Section III. This provides an optimal deformation between $f_{U S}$ and $f_{M R I}$ under the elastic metric and the shooting vector field on $f_{U S}$ (we always use $f_{U S}$ as the source surface and $f_{M R I}$ as the target surface). We then define a function $H:[0,1] \rightarrow \mathbb{R}_{+}$as $H(v)=\int_{u \in \mathbb{S}^{1}}\left|F_{t}^{*}(0)(u, v)\right|^{2} d u$, where $\dot{F}_{t}^{*}(0)$ is the initial velocity vector of the geodesic path from $f_{U S}$ to the re-parameterized $f_{M R I}$. We remind the reader that the shooting vector is sufficient to characterize the geodesic path or the deformation from one shape to the other. Let $v^{*}=\operatorname{argmin}_{v \in[0,1]} H(v)$. The quantity $H\left(v^{*}\right)$ represents our estimate of a quantification of the elasticity of the ultrasound surface. We also use $v^{*}$ to find the curve on $f_{M R I}$ that most corresponds to $\beta_{U S}$, as follows. Select the curve on $f_{M R I}$ that registers with $f_{U S}\left(\cdot, v^{*}\right)$ under the optimal registration obtained above and call it $\beta_{M R I}$. It is important to note that $\beta_{M R I}$ can be non-planar. This is a reasonable possibility because the corresponding transformation to optimally register the ultrasound and MRI images is often nonlinear.

Figure 7 provides a depiction of the entire pipeline of our framework and presents results for one of the subjects in our study. In this case, we found that the measure of elasticity of the endometrial tissue was given by $H\left(v^{*}\right)=13.54$ (it corresponds to the red point in Figure 7(a)). 


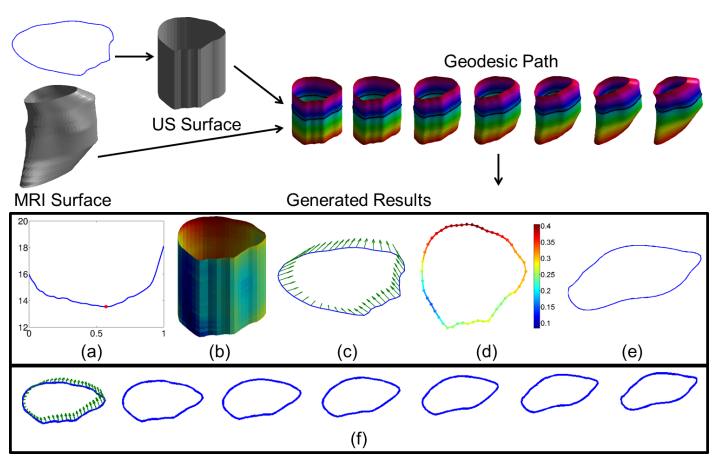

Fig. 7. Pipeline of our framework. The curve $\beta_{U S}$ leads to a generalized cylinder $f_{U S}$ that is registered and deformed into $f_{M R I}$ using elastic shape geodesic. The geodesic provides (a) $H(v)$, the magnitude of the shooting vector field at each level of the US surface, with its minimum value $H\left(v^{*}\right)$ marked using a red dot, (b) a visualization of the magnitude of this vector field on $f_{U S}$ (hot colors correspond to higher magnitude), (c) the deformation vector field on the selected $\beta_{U S}$ and (d) its magnitude, (e) the optimal curve $\beta_{M R I}$ on the MRI surface, and (f) the deformation between the $\beta_{U S}$ and $\beta_{M R I}$ curves.

\section{EXPERIMENTAL RESULTS}

Now we apply our framework on real data to estimate the quantities of interest $-\beta_{M R I}$, elasticity measure $H\left(v^{*}\right)$, etc. and to achieve the stated goals. However, since an important part of the experimentation is the evaluation of results, we are interested in quantifying our estimation performance. The difficulty in this evaluation comes from the fact that the ground truth is unknown. It is even difficult to visually evaluate the registration due to the complexity introduced by different dimensions (2D versus 3D) and different modalities (TVUS versus MRI). Therefore, it is important to generate some semisynthetic data where the ground truth is known by construction and one can easily compare the estimated quantities with the ground truth. In terms of evaluating inferences on real data, we will rely on annotations made by the experts, for $\beta_{M R I}$, and compare them with the estimated $\beta_{M R I}$. These experimental results and their evaluations are described next.

\section{A. Validation on Semi-Synthetic Data}

As stated earlier, the non-rigid deformation $\Phi$ is due to the transducer's pressure and, thus, the deformation location and magnitude depend on: 1) the position of the transducer, especially the surface of contact between the transducer and the organ; 2) the magnitude and the direction of its pressure. In order to imitate the real-world process, we choose an elastic deformation model that follows these physical principles and generate a controlled deformation as follows:

1) Pick up a random point on the surface around which we define a contact area between the transducer and the organ.

2) Apply different strengths of deformations, ranging from weak to strong, controlled by the deformation model.

3) Choose random directions to simulate the direction of pressure.

Once the original surface $f_{M R I}$ is deformed, using one of the deformation fields $\Phi$ described above, we randomly choose

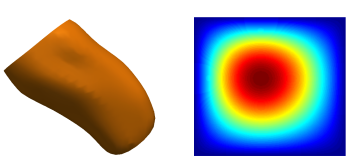

(a)

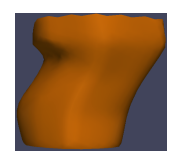

(c)

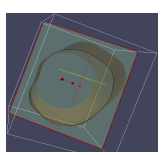

(d)
Fig. 8. An illustration of semi-synthetic data. (a) $f_{M R I}$, (b) a deformation vector field generating $\Phi$, (c) the deformed surface $\Phi\left(f_{M R I}\right)$, and (d) a cutting plane $P$ resulting in $\beta_{U S}$.

a point on the deformed surface $\Phi\left(f_{M R I}\right)$ and a normal vector to define an intersecting plane $P$. The intersection $P \cap \Phi\left(f_{M R I}\right)$ is a planar curve $\beta_{U S}$, which plays the role of a manually extracted curve from the TVUS image. Fig. 8 illustrates the experimental design for the construction of synthetic deformations, for which the correspondence between surfaces is known. In the process, we generate an estimate $\hat{\beta}_{M R I}$ of $\beta_{M R I}$ on the original surface $f_{M R I}$. An example of geodesic paths between the original MRI-surfaces and the reconstructed TVUS-surfaces is given in Fig. 9.

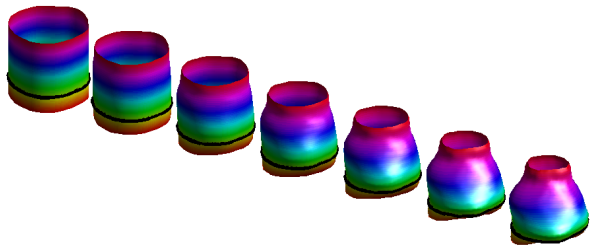

Fig. 9. The geodesic between $f_{U S}$ and $f_{M R I}$ surfaces, with the estimated curve $\hat{\beta}_{M R I}$ marked in black on $f_{M R I}$.

Since we have the original curve $\beta_{M R I}$ and its estimate $\hat{\beta}_{M R I}$, we can quantify the estimation performance quite naturally. In the top panel of Fig. 10, we present an evaluation of the differences between these curves for ten simulated cases under four different metrics - root-mean squared (RMS or STD), supremum norm, and fiducial registration error (FRE). As we can see, especially using the supremum norm, the error between the curves is very small in most of the cases and our framework is able to estimate $\beta_{M R I}$ faithfully. For comparison purposes, we have applied the same experimental protocol to register $f_{M R I}$ and $f_{U S}$ using an ICP-based approach and present these results in the bottom panel of Fig. 10. Our approach significantly outperforms this method.

\section{B. Qualitative Validation}

We start by displaying results of geodesic deformations between two surfaces in Fig. 11. In each example, we display the geodesic path, and the deformation between the ultrasound curve and the optimal MRI curve chosen using our method. The correspondence of points across surfaces is mapped onto the whole geodesic where points corresponding to each other are shaded with the same colors. In addition, we plot the optimal curves along the geodesic path in black color.

As a main qualitative approach to validation of our estimation, we investigate results on real data from ten patients. As described earlier, we obtained $f_{M R I}$ and $\beta_{U S}$ for each of these cases using expert segmentation and used them to 

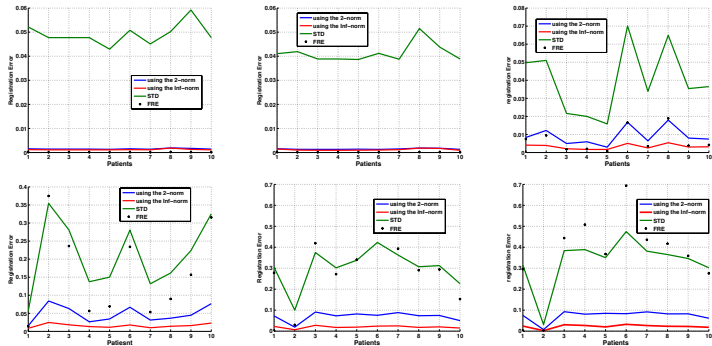

Fig. 10. Estimation error between $\beta_{M R I}$ and $\hat{\beta}_{M R I}$ for ten examples using semi-synthetic data under several metrics (FRE (black), RMS (green), $L^{\infty}$ (red), and $L^{2}$ (blue)) using our method in the first row and an ICP-based method in the second row. Columns range from weak deformations (left) to strong deformations (right).

(a)
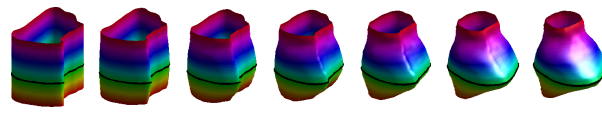

(b)

(a)
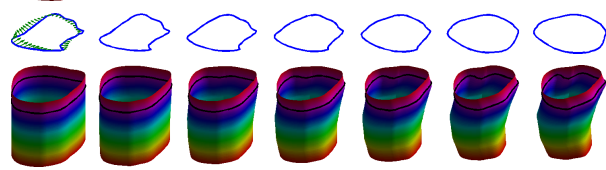

(b)

Fig. 11. For two different patients, we display (a) the geodesic between US and MRI surfaces, with the optimal curve marked in black, and (b) the corresponding deformation between curves with the deformation vector field on the US curve.

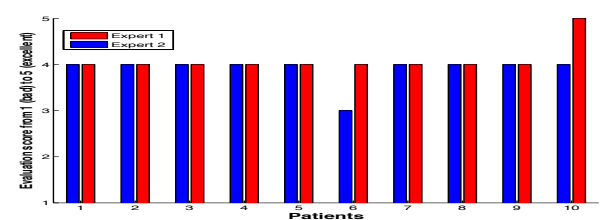

Fig. 12. Qualitative evaluation by two experts. For each patient, the performance is evaluated by a score from 1 (bad) to 5 (excellent).

compute the estimate $\hat{\beta}_{M R I}$. In this case, the ground truth $\left(\beta_{M R I}\right)$ is not available and we asked two experts to evaluate the results. The experts were shown the estimated curve $\hat{\beta}_{M R I}$, the deformation between $\beta_{U S}$ and the estimated curve, and a plane that contains as much of $\hat{\beta}_{M R I}$ as possible. The evaluation is given in Fig. 12, and examples of $H(v)$ (with $H\left(v^{*}\right)$ highlighted in red) and the estimated magnitude of the vector field on $\beta_{U} S$ are shown in Fig. 13.

(a)

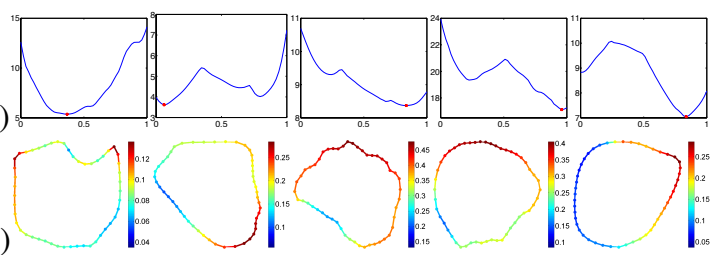

Fig. 13. Estimation of $\hat{\beta}_{M R I}$ : for each patient, we display (a) $H(v)$ and (b) the corresponding magnitude of the deformation vector field on $\beta_{U S}$
To evaluate the performance of the proposed method, we setup a registration quality score ranging from 1 (bad) to 5 (excellent). The experts rated eight out of ten as 4 , one as 3 , and one as 5 - a very high evaluation of the estimation performance. We note that the experts' evaluation is based on the anatomical features, tissue structures, and the surrounding organs, like the uterus. We observe that the deformation magnitude provides a nice display of elasticity along the curve. Such information is very useful when coupled with the localization of the tissue (surrounding organs) as it will help a surgeon evaluate the phase of the disease and the tissue's mobility.

\section{DisCUSSION}

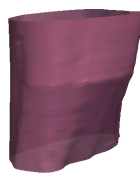

(a)

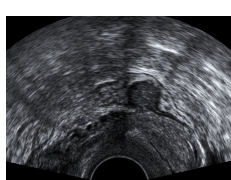

(b)

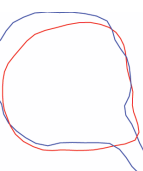

(c)

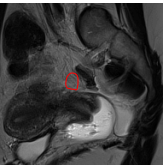

(d)
Fig. 14. (a) The MRI surface, (b) the TVUS image, (c) the overlap between $\beta_{U S}$ from (b) and the estimated curve $\hat{\beta}_{M R I}$ on (a), and (d) $\hat{\beta}_{M R I}$ on the estimated intersecting plane $P$.

Since our approach provides the optimal curve $\hat{\beta}_{M R I}$ on the MRI surface that matches the original TVUS curve, we can locate $\hat{\beta}_{M R I}$ in the MRI volume. In Fig. 14, we show this result on one patient. Given the segmented MRI surface $f_{M R I}$ (Fig. 14(a)) and the TVUS image (Fig. 14(b)) we describe the clinical utility of our approach as following: first, we have constructed $f_{U S}$ and then applied our approach to register them and to estimate $\hat{\beta}_{M R I}$ on $f_{M R I}$; second, we have computed its barycentre and its normal to define the cutting plane $P$; third, we have interpolated the corresponding plane $P_{M R I}$ between the MRI volume and $P$; finally, we have projected $\hat{\beta}_{M R I}$ on $P_{M R I}$ with small changes to show its closest position on $P_{M R I}$ (Fig. 14(d)). To show the performance of the registration, Fig. 14(c) displays the overlap of the original curve $\beta_{U S}$ and the estimated curve $\hat{\beta}_{M R I}$.

As stated in the introduction, our clinical goals in this work were to combine complementary information from MRI and TVUS (transvaginal ultrasound) images of rectovaginal endometriotic nodules, to locate the US intersecting plane in the MRI volume as shown in Fig. 14(d), and to quantify the deformability of endometrial tissues and surrounding structures as shown in Fig. 13. These goals were reached using two models: (1) a clinical model from TVUS and MRI images (10 patients) and (2) a semi-synthetic model (original data were deformed using a biomechanical model to simulate physical deformations). A segmentation (delimitation) of the nodule was performed in the two modalities (MRI and TVUS) and a recent shape analysis approach was applied, with appropriate modifications. The study resulted in an accurate quantification and localization of the deformable endometriotic tissues.

In short, using both semi-synthetic simulated data and the original real data from ten patients, we were able to visually 
improve the preoperative diagnosis by facilitating interpretation of ultrasound data. Specifically, combining images from MRI and TVUS allows us to compensate for differences in patient positioning during MRI and TVUS observations, and the potential deformation of endometrial tissue during the TVUS measurement process (transducer's pressure). An immediate impact of these results will be in improving the planning of surgical procedures. Furthermore, we believe that combining complementary information from both modalities, by locating the US plane inside the MRI volume, and quantifying deformability of endometriosis under a given level of applied stress can open new avenues for diagnosing and monitoring complex cases.

Nevertheless, there are some limitations in the performed study. One is the use of boundaries that were manually extracted by the experts to represent the true shape of the endometrial tissue. As a consequence, the deformation vector field depends on the quality of the segmentation. This can introduce a bias when the quality of the imaging modalities decreases or when the endometrial tissues are present in very small areas. Another application of this methodology (with sufficient adaptation to the new problem) can be the registration of cylindrical representations of the colon in different modalities to prevent the development of advanced colorectal cancer. Such study will allow practitioners to quantify the uncontrolled cell growth in the colon or rectum that may cause colorectal cancer.

\section{SUMMARY}

In this work, we have proposed an elastic shape analysis framework for 2D-3D TVUS-MR registration to localize and characterize endometrial tissues. The information is available in the form of parametrized surfaces in the 3D MRI data and segmented closed curves in the 2D TVUS images. Our approach extrapolates the US curve into a generalized cylinder and applies an elastic shape analysis technique to obtain registration and deformation between the two surfaces. This way, we obtain the optimal curve on the MRI surface that corresponds to the original TVUS curve. We also obtain deformations and quantification of the deformations as measures of elasticity at different regions on the tissue.

Using both semi-synthetic and real data, we are able to validate the estimation results and demonstrate the appropriateness of elastic shape analysis in this context. In the semi-synthetic data, the closeness of estimated quantities (curves and deformations) with the ground truth underscore the success of this framework despite a loss of information inherent in the 2D nature of US imaging. A qualitative evaluation by two experts, that involves more syntactical information, generally rated the estimation results from very good to excellent.

\section{ACKNOWLEDGEMENTS}

Anuj Srivastava was supported in part by the NSF DMS 1208959 and Chafik Samir was supported in part by Auvergne Regional Council grants.

\section{REFERENCES}

[1] L. Chamie, R. Blasbalg, R. Pereira, G. Warmbrand, and P. Serafini, "Findings of pelvic endometriosis at Transvaginal US, MR imaging, and Laproscopy," RadioGraphics, vol. 31, pp. 71-100, 2011.

[2] A. Kennedy and C. Peterson, "Transvaginal sonography in reproductive endocrinology and infertility," Reproductive Endocrinology and Infertility, pp. 545-565, 2010.

[3] J. Modersitzki, "Regularization," in FAIR: Flexible Algorithms for Image Registration, 2009, pp. 117-135.

[4] E. G. Learned-Miller and P. Ahammad, "Joint mri bias removal using entropy minimization across images," in NIPS, 2004.

[5] T. Kadir and M. Brady, "Saliency, scale and image description," International Journal of Computer Vision, vol. 45, no. 2, pp. 83-105, 2001.

[6] T. Hopp, M. Dietzel, P. Baltzer, P. Kreisel, W. Kaiser, H. Gemmeke, and N. Ruiter, "Automatic multimodal $2 \mathrm{~d} / 3 \mathrm{~d}$ breast image registration using biomechanical fem models and intensity-based optimization," Medical Image Analysis, 2012.

[7] J. H. Hipwell, G. P. Penny, R. A. McLaughlin, K. Rhode, P. Summers, T. C. Cox, J. V. Byrne, J. A. Noble, and D. J. Hawkes, "Intensity-based 2d-3d registration of cerebral angiograms," IEE Trans. Medical Imaging, vol. 22, no. 11, 2003.

[8] J. B. A. Maintz, P. A. van den Elsen, and M. A. Viergever, "Registration of 3d medical images using simple morphological tools," in IPMI, 1997, pp. 204-217.

[9] A. Andronache, M. von Siebenthal, G. Székely, and P. Cattin, "Nonrigid registration of multi-modal images using both mutual information and cross-correlation," Medical Image Analysis, vol. 12, no. 1, pp. 3 $15,2008$.

[10] A. Roche, X. Pennec, G. Malandain, and N. Ayache, "Rigid registration of $3 \mathrm{~d}$ ultrasound with $\mathrm{mr}$ images: A new approach combining intensity and gradient information," IEEE Trans. Med. Imaging, vol. 20, no. 10 pp. 1038-1049, 2001.

[11] A. Cosse, "Diffeomorphic surface-based registration for mr-us fusion in prostate brachytherapy," in Electrotechnical Conference (MELECON), 2012 16th IEEE Mediterranean, vol. 1, 2012, pp. 903 -907.

[12] M. Vermandel, N. Betrouni, D. Pasquier, J. Gauvrit, C. Vasseur, and J. Rousseau, "A $2 \mathrm{~d} / 3 \mathrm{~d}$ matching based on a hybrid approach: improvement to the imaging flow for avm radiosurgery," IEEE Eng Med Biol Soc, vol. 3, 2005 .

[13] A. Biesdorf, K. Rohr, D. Feng, H. von Tengg-Kobligk, F. Rengier, D. Backler, H.-U. Kauczor, and S. Warz, "Segmentation and quantification of the aortic arch using joint $3 \mathrm{~d}$ model-based segmentation and elastic image registration," Medical Image Analysis, vol. 16, no. 6, pp. $1187-1201,2012$.

[14] D. Zikic, B. Glocker, O. Kutter, M. Groher, N. Komodakis, A. Kamen, N. Paragios, and N. Navab, "Linear intensity-based image registration by markov random fields and discrete optimization," Medical Image Analysis, vol. 14, no. 4, pp. 550 - 562, 2010.

[15] C. Buerger, T. Schaeffter, and A. P. King, "Hierarchical adaptive local affine registration for fast and robust respiratory motion estimation," Medical Image Analysis, vol. 15, no. 4, pp. 551-564, 2011.

[16] C. Zhang, G. E. Christensen, S. Kurtek, A. Srivastava, M. J. Murphy, E. Weiss, E. Bai, and J. Williamson, "Supir: Surface uncertaintypenalized, non-rigid image registration for pelvic ct imaging," in Biomedical Image Registration, vol. 7359, 2012, pp. 236-245.

[17] S. Kurtek, E. Klassen, J. Gore, Z. Ding, and A. Srivastava, "Elastic geodesic paths in shape space of parametrized surfaces," IEEE Trans. Pattern Analysis and Machine Intelligence, vol. 34, no. 9, pp. 1717$1730,2012$.

[18] S. Kurtek, E. Klassen, Z. Ding, S. Jacobson, J. Jacobson, M. Avison, and A. Srivastava, "Parameterization-invariant shape comparisons of anatomical surfaces," IEEE Trans. Medical Imaging, vol. 30, no. 3, pp. $849-858,2011$

[19] I. Jermyn, S. Kurtek, E. Klassen, and A. Srivastava, "Elastic shape matching of parameterized surfaces using square root normal fields," in European Conference on Computer Vision, 2012, pp. 804-817.

[20] S. Kurtek, E. Klassen, Z. Ding, and A. Srivastava, "A novel Riemannian framework for shape analysis of 3D objects," in Proc. IEEE Computer Vision and Pattern Recognition, 2010, pp. 1625-1632.

[21] X. Gu and B. C. Vemuri, "Matching 3D shapes using 2D conformal representations," in Proc. of MICCAI, vol. 3218, 2004, pp. 771-780. 